\title{
Rapid visualization of latent fingermarks using gold seed-mediated enhancement
}

(a) CrossMark

\author{
Chia-Hao Su ${ }^{1,2}$, Chun-Chieh Yu ${ }^{1}$ and Fong-Yu Cheng ${ }^{3 *}$
}

\begin{abstract}
Background: Fingermarks are one of the most important and useful forms of physical evidence in forensic investigations. However, latent fingermarks are not directly visible, but can be visualized due to the presence of other residues (such as inorganic salts, proteins, polypeptides, enzymes and human metabolites) which can be detected or recognized through various strategies. Convenient and rapid techniques are still needed to provide obvious contrast between the background and the fingermark ridges and to then visualize latent fingermark with a high degree of selectivity and sensitivity.
\end{abstract}

Results: In this work, lysozyme-binding aptamer-conjugated Au nanoparticles (NPs) are used to recognize and target lysozyme in the fingermark ridges, and $\mathrm{Au}^{+}$-complex solution is used as a growth agent to reduce $\mathrm{Au}^{+}$from $\mathrm{Au}^{+}$ to $\mathrm{Au}^{0}$ on the surface of the Au NPs. Distinct fingermark patterns were visualized on a range of professional forensic within 3 min; the resulting images could be observed by the naked eye without background interference. The entire processes from fingermark collection to visualization only entails two steps and can be completed in less than 10 min. The proposed method provides cost and time savings over current fingermark visualization methods.

Conclusions: We report a simple, inexpensive, and fast method for the rapid visualization of latent fingermarks on the non-porous substrates using Au seed-mediated enhancement. Au seed-mediated enhancement is used to achieve the rapid visualization of latent fingermarks on non-porous substrates by the naked eye without the use of expensive or sophisticated instruments. The proposed approach offers faster detection and visualization of latent fingermarks than existing methods. The proposed method is expected to increase detection efficiency for latent fingermarks and reduce time requirements and costs for forensic investigations.

Keywords: Aptamers, Au nanoparticles, Au seeds, Fingermarks, Lysozyme

\section{Background}

Fingermarks are deposited when the ridged skin surface of a finger touches an object and creates an imprint on the object's surface $[1,2]$. The unique patterns of fingermarks make them become one a key form of physical evidence in forensic investigations [2-4]. When imprinted on opaque media such as paint, fingermarks are directly visible to the naked eye. However, latent fingermarks are not visible to the naked eye and can only be detected through visualizing certain residues (such as amino acids and lipids) present within the fingermark [5-7]. The

\footnotetext{
*Correspondence: zfy3@ulive.pccu.edu.tw

${ }^{3}$ Department of Chemistry, Chinese Culture University, 55, Hwa-Kang

Road, Yang-Ming-Shan, Taipei 11114, Taiwan

Full list of author information is available at the end of the article
}

rapid and reliable visualization of latent fingermarks is important to help police quickly identify potential suspects by comparing crime-scene fingermarks against existing fingermark databases. Currently common methods include the use of carbon powder, cyanoacrylate or triketohydrindene hydrate. Carbon powder physically adsorbs the latent fingermarks, while cyanoacrylate and triketohydrindene hydrate react with the protein's primary amine to visualize latent fingermarks. Recently, various optical, chemical and physical techniques based on nanomaterials have been developed to provide obvious contrast between the background and the fingerprint ridges [8-10].

Recently, studies of specific biomolecule-targeting and nanomaterials have raised the possibility of increasing 
selectivity and sensitivity in fingermark [11-13]. Wood et al. reported a highly selective technique using a lysozyme targeting-aptamer-based reagent to visualize latent fingermarks with fluorescence images [14]. Shan et al. [15] and $\mathrm{Xu}$ et al. [16] respectively combined electrochemistry with surface plasmon resonance (SPR) and enzyme immunoassay to detect fingermarks. $\mathrm{Li}$ et al. used the SPR of aptamer-tagged Au nanoparticles (NPs) to visualize fingermarks $[17,18]$. Very recently, He et al. developed a simple method termed immunological multimetal deposition (iMMD) which combines immunoassay and conventional MMD to allow for the naked-eye visualization of sweat fingermarks using silver staining [19]. The iMMD method requires fewer steps and less time than conventional and improved MMD. However, background interference can negatively impact the visual enhancement of the fingermark ridges during the silver staining processes. In practice, the concentrations and incubation times of silver staining are difficult to calculate and control because of the unknown quantity of $\mathrm{Au}$ NPs in the fingermarks. For the silver staining solution, increased incubation time will produce an obvious background, thus reducing the visual contrast of the ridges.

To reduce the impact of background interference in techniques such as silver-staining, and to improve on the specific targeting of fingermarks in other methods based on the use of carbon powders, cyanoacrylate or triketohydrindene, this paper combines aptamer and metalstaining strategies [14, 20-22] for the rapid naked-eye visualization of latent fingermarks using a Au seed-mediated growth technique. In this technique, $\mathrm{Au}$ seed-mediated growth is a highly efficient chemical strategy for the synthesis of monodispersed nanoparticles. Au seed is as a nucleation center and can catalyze the reduction of $\mathrm{Au}^{+}$ ions at room temperature without the use of reducing agents. This reduction from $\mathrm{Au}^{+}$to $\mathrm{Au}^{0}$ is an autocatalytic process and takes place on the surface of Au seeds. Scheme 1 shows a schematic representation of the proposed method. The process entails two main steps, and takes less than $10 \mathrm{~min}$ in total. We chose lysozyme as the targeted molecule because it is found in abundance in fingermark residues [20-22]. For the sensitive and selective targeting of lysozyme, Au seeds were conjugated with lysozyme-binding aptamers (LBA, 5'-thiol (SH)-TTTT TTATCAGGGCTAAAGAGTGCAGAGTTACTTAG-3') $[17,18]$ by a covalent bond ( $\mathrm{Au}-\mathrm{S}$ bond). Thus, LBAconjugated $\mathrm{Au}(\mathrm{LBA}-\mathrm{Au})$ seeds can specifically recognize and target lysozyme in ridges. When LBA-Au seeds are treated with $\mathrm{Au}^{+}$-complex solution, concentrations of $\mathrm{Au}^{+}$ions could be rapidly reduced and grown on the seed surface, resulting in larger Au NPs through a self-catalysis process (from $\mathrm{Au}^{+}$to $\mathrm{Au}^{0}$ ) of the $\mathrm{Au}$ seeds. The large $\mathrm{Au}$ NPs are red in color, thus the unique patterns of latent fingerprints are rapidly visualized and can be observed by the naked eye in a short time (i.e., less than $10 \mathrm{~min}$ ).

\section{Methods}

Preparation of tetrakis(hydroxypropyl)phosphonium chloride-stabilized Au (THPC-Au) NPs

THPC-Au NPs were prepared as previously described [23]. First, the THPC solution was prepared by diluting $12 \mu \mathrm{L}$ of $80 \%$ THPC solution to $1 \mathrm{~mL}$ with deionized water. Subsequently, $1 \mathrm{~mL}$ of the as-prepared THPC solution, $0.5 \mathrm{~mL}$ of $\mathrm{NaOH}(1 \mathrm{M})$, and $45 \mathrm{~mL}$ of deionized water were vigorously stirred in a flask at room temperature for $5 \mathrm{~min} .10 \mathrm{~mL}$ of $\mathrm{HAuCl}_{4}(5 \mathrm{mM})$ was then added. Once the color of the solution turned from light yellow to dark brown, indicating the formation of THPC-Au NPs, the mixture was stirred for another $2 \mathrm{~h}$. THPC-Au NP solution was stored at $4{ }^{\circ} \mathrm{C}$ for further use. The average size of the as-prepared THPC-Au NPs was calculated using transmission electron microscopy (TEM, H-7500; Hitachi Koki Co., Tokyo, Japan). Electron micrographs of THPC-Au NPs and LBA-Au NPs were obtained by placing a drop of the sample onto a copper mesh coated with an amorphous carbon film and dried in a vacuum desiccator.

The particle concentration of THPC-Au NPs was based on the Au ion concentration measured using inductivelycoupled plasma (ICP) analysis to calculate as $\mu \mathrm{M}$. Au ion concentrations were measured by ICP in terms of $\mathrm{mg} / \mathrm{L}$ and the concentration could be converted to mmole/ $\mathrm{L}$. The average size of THPC-Au NPs could then be determined via TEM imaging. We assumed Au NPs were spherical structures to calculate the volume of a single $\mathrm{Au}$ sphere, and the volume of a single Au atom could be calculated based on its diameter. Dividing the volume of one $\mathrm{Au}$ NP by the volume of one Au atom obtains the number of $\mathrm{Au}$ atoms contained in a single $\mathrm{Au}$ NP. We assumed that $\mathrm{N} \mathrm{Au}$ atoms could form one Au NP. Therefore, the particle concentration $(\mu \mathrm{M})$ of THPC-Au NPs could be calculated after dividing the $\mathrm{Au}$ ion concentration $(\mu \mathrm{M})$ by $\mathrm{N}$. The particle concentration of as-prepared THPCAu NPs was $2.64 \mu \mathrm{M}$. 


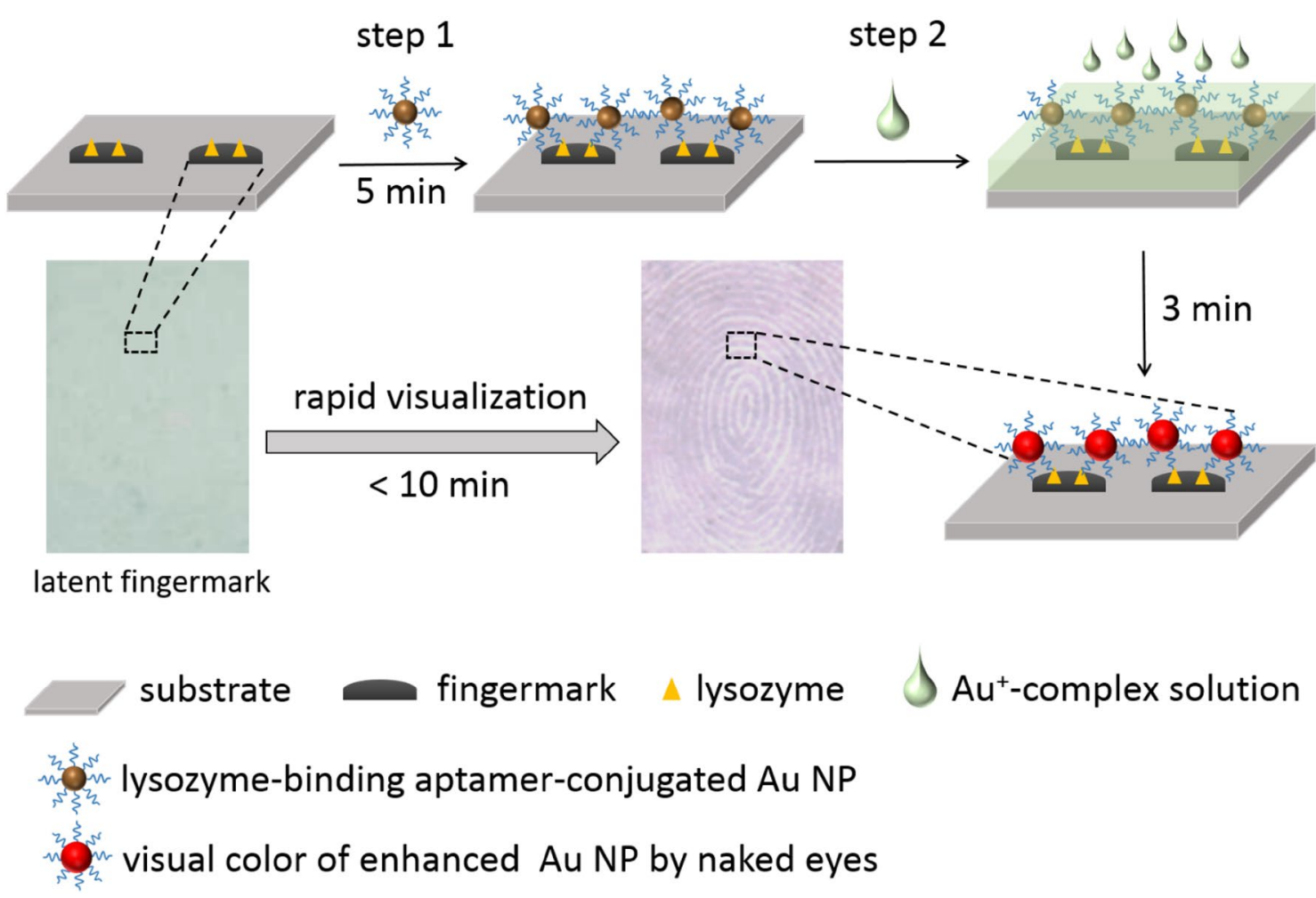

Scheme 1 Schematic representation of rapid visualization of latent fingermarks by Au seed-mediated enhancement technique. Lysozyme-binding aptamer-conjugated Au NPs specifically recognize and target lysozyme in latent fingermarks (step 1). After treating with $\mathrm{Au}^{+}$-complex solution (step 2), bigger Au NPs with visible color are produced from Au seeds and can be clearly seen with the naked eye within 3 min. The total process from fingermarks collection to visualization can be completed within 10 min

\section{Preparation of lysozyme-binding aptamer (LBA)-conjugated THPC-Au (LBA-Au) NPs}

Thiol (SH)-modified LBAs (HS-LBA, 5'-HS-TTTT TTATCAGGGCTAAAGAGTGCAGAGTTACTTAG-3') were used to prepare LBA-Au NPs. First, $26.4 \mu \mathrm{L}$ of HSLBA was mixed with $873.6 \mu \mathrm{L}$ of deionized water, and then $100 \mu \mathrm{L}$ of THPC-Au NPs (particle concentration: $2.64 \mu \mathrm{M})$ was added to the mixture and the solution was stirred for $4 \mathrm{~h}$. The LBA-Au NPs (particle concentration: $0.264 \mu \mathrm{M})$ could then be directly used without further purification.

However, in experiments for the specific targeting and selection of lysozyme for LBA-Au NPs, Hex-LBA-Au NPs were prepared by replacing HS-LBA-Hex with HS-LBA. Hex-LBA-Au NPs were purified using a high-speed centrifuge at $100,000 \mathrm{~g}$ for $10 \mathrm{~min}$ to remove free Hex-LBA in the supernatant. The precipitate was redispersed and stored in deionized water at $4{ }^{\circ} \mathrm{C}$ for further use.

\section{Preparation of $\mathrm{Au}^{+}$-complex solution}

Cetyltrimethylammonium bromide (CTAB, $0.182 \mathrm{~g}$ ) was dissolved in $9 \mathrm{~mL}$ of deionized water before adding $1 \mathrm{~mL}$ of $\mathrm{HAuCl}_{4}(5 \mathrm{mM})$. Subsequently, $60 \mu \mathrm{L}$ of ascorbic acid (100 mM) was added to the mixture. The color of the solution changing from dark orange to colorless limpidity indicated the $\mathrm{Au}^{+}$-complex solution $\left(\left[\mathrm{Au}^{+}\right]=470 \mu \mathrm{M}\right)$ was complete and ready for further use.

\section{Characterization}

The absorption spectrum of the nanomaterials and biomolecules were determined using an ultraviolet-visible (UV-vis) spectrophotometer (HP8453; Agilent Technologies, Santa Clara, CA, USA).

\section{Test of Au seed-mediated enhancement}

First, $997.5 \mu \mathrm{L}$ of the $\mathrm{Au}^{+}$-complex solution was added to $1 \mathrm{~mL}$ of eppendorf, followed by $2.5 \mu \mathrm{L}$ of THPC-Au 
NPs (particle concentration: $2.64 \mu \mathrm{M}$ ). Following the mixing of the $\mathrm{Au}^{+}$-complex solution and THPC-Au NPs, the seed growth reaction process was monitored using a $\mathrm{UV}$-vis spectrophotometer at intervals of $10 \mathrm{~s}$ for $5 \mathrm{~min}$.

\section{Collection of fingermarks}

Professional-grade forensic tape was used to collect fingermarks. Volunteers first washed their fingers with soap and water and then dried them in air before pressing their fingertips on the tape. Fingermarks were collected immediately following deposition and after a 24 delay for further tests.

\section{Rapid visualization of latent fingermarks}

First, $200 \mu \mathrm{L}$ of LBA-Au NPs (particle concentration: $132 \mathrm{nM}$ ) were spread over the fingermarks on the substrate. After incubating for $5 \mathrm{~min}$, the sample was rinsed two times with deionized water to remove free LBA-Au NPs. Next, $200-300 \mu \mathrm{L}$ of the $\mathrm{Au}^{+}$-complex solution was spread over the fingermark region, followed by incubation for 2-3 min. Notably, the required visualization time of the latent fingermarks was based on the quantity of lysozyme from different volunteers. In our experimental tests, the time required to visualize latent fingermarks did not exceed $5 \mathrm{~min}$.

\section{Results and discussion}

In this study, the tetrakis(hydroxypropyl)phosphonium chloride-stabilized Au (THPC-Au) NPs were chosen as $\mathrm{Au}$ seeds. THPC-Au NPs were prepared by the chemical reduction method described by Pham et al. [23]. The diameter of as-prepared THPC-Au NPs is $\sim 2.6 \mathrm{~nm}$. Subsequently, thiol-modified LBAs were conjugated with THPC-Au NPs using a covalent bond of $\mathrm{Au}-\mathrm{S}$. Figure 1a, b respectively show the transmission electron microscopy (TEM) images and size distribution histogram of THPC-Au NPs. Figure 1c, d respectively show the transmission electron microscopy (TEM) images and size distribution histogram of LBA-conjugated THPC-Au (LBA-Au) NPs. The TEM morphologies of THPC-Au NPs and LBA-Au NPs are identical, indicating that LBAs have no influence after being conjugated to the surface of THPC-Au NPs. Figure 1e shows the absorbance spectra of the LBAs, THPC-Au NPs, and LBA-Au NPs. LBAs have a characteristic absorption band at $\sim 260 \mathrm{~nm}$ contributed from oligonucleotides. THPC-Au had a weak absorption band $\sim 510 \mathrm{~nm}$ and the weak absorption is due to their smaller size. As seen in the spectrum of LBA-Au NPs, two characteristic bands of $\sim 260$ and $\sim 510 \mathrm{~nm}$ are observed, indicating that LBAs are conjugated on the $\mathrm{Au}$
NPs. To confirm the lysozyme binding ability and selectivity of LBA-Au NPs, selective tests of lysozyme were designed on a glass microscope slide and monitored by fluorescence images (Fig. 2). Figure 2 shows the results of lysozyme-selection test of LBA-Au NPs. The PBS-treated region (the left region in Fig. 2) had no fluorescence after treatment with LBA-THPC-Au NPs. This indicates that LBA-THPC-Au NPs have no non-specific binding to the glass microscope slide. In the bovine serum albumin (BSA) region (the middle region in Fig. 2), no fluorescence was observed after treatment with LBA-Au NPs. This was due to LBA-Au NPs being unable to bind to BSA and they were later removed by washing with deionized water. As seen in lysozyme region (the right region in Fig. 2), after treatment with LBA-Au NPs for $5 \mathrm{~min}$, fluorescence could be clearly observed. These tested results indicate that LBA-Au NPs could maintain the specific targeting function and selectivity of lysozyme after LBAs are conjugated on the surface of THPC-Au NPs.

To achieve the Au seed-mediated visualization of latent fingermarks, $\mathrm{Au}^{+}$-complex solution also played an essential role. The $\mathrm{Au}^{+}$-complex solution was composed of gold(III) chloride hydrate $\left(\mathrm{HAuCl}_{4}\right)$, cetyltrimethylammonium bromide (CTAB), and ascorbic acid (AA). The preparation of the $\mathrm{Au}^{+}$-complex solution involves two steps. The first step mixes $\mathrm{HAuCl}_{4}$ and CTAB in the aqueous solution to produce $\mathrm{Au}^{3+}{ }_{-} \mathrm{CTAB}$ complexes [24-27]. The second step adds AA to reduce $\mathrm{Au}^{3+}{ }_{-} \mathrm{CTAB}$ complexes to $\mathrm{Au}^{+}-\mathrm{CTAB}$ complexes. In the presence of $\mathrm{CTAB}, \mathrm{AA}$ cannot directly reduce $\mathrm{Au}^{3+}$ to $\mathrm{Au}^{0}[28,29]$. Importantly, the $\mathrm{Au}^{+}-\mathrm{CTAB}$ complexes only can by stable in an aqueous solution without Au seeds. Because $\mathrm{Au}^{+}-\mathrm{CTAB}$ complexes can be rapidly reduced to $\mathrm{Au}^{0}$ in the presence of $\mathrm{Au}$ seeds in a reduction reaction which does not require an additional reducing agent [24-29]. The reduction process of $\mathrm{Au}^{+}-\mathrm{CTAB}$ complexes occurred on the $\mathrm{Au}$ seed surface and the reduced $\mathrm{Au}^{0}$ directly grew on the seed surface resulting in particle growth of $\mathrm{Au}$ seeds, rapidly producing larger sized and visually colourful Au NPs. Thus, this Au seed-mediated enhancement system is very sensitive to the presence of Au seeds in the solution. Figure 3a shows the tested results of Au seedmediated growth for THPC-Au NPs incubated with $\mathrm{Au}^{+}$complex solution with a ratio $\left(\left[\mathrm{Au}^{+}\right] /[\mathrm{Au}\right.$ seeds] $\left.]\right)$ of 152 . The color of solution changed from limpid to bright red within 1 min after incubation with THPC-Au NPs and the $\mathrm{Au}^{+}$-complex solution. This color change indicated that the THPC-Au seeds became larger Au NPs through a seed growth process. Figure $3 \mathrm{~b}, \mathrm{c}$ respectively show the TEM image and size distribution histogram of the 

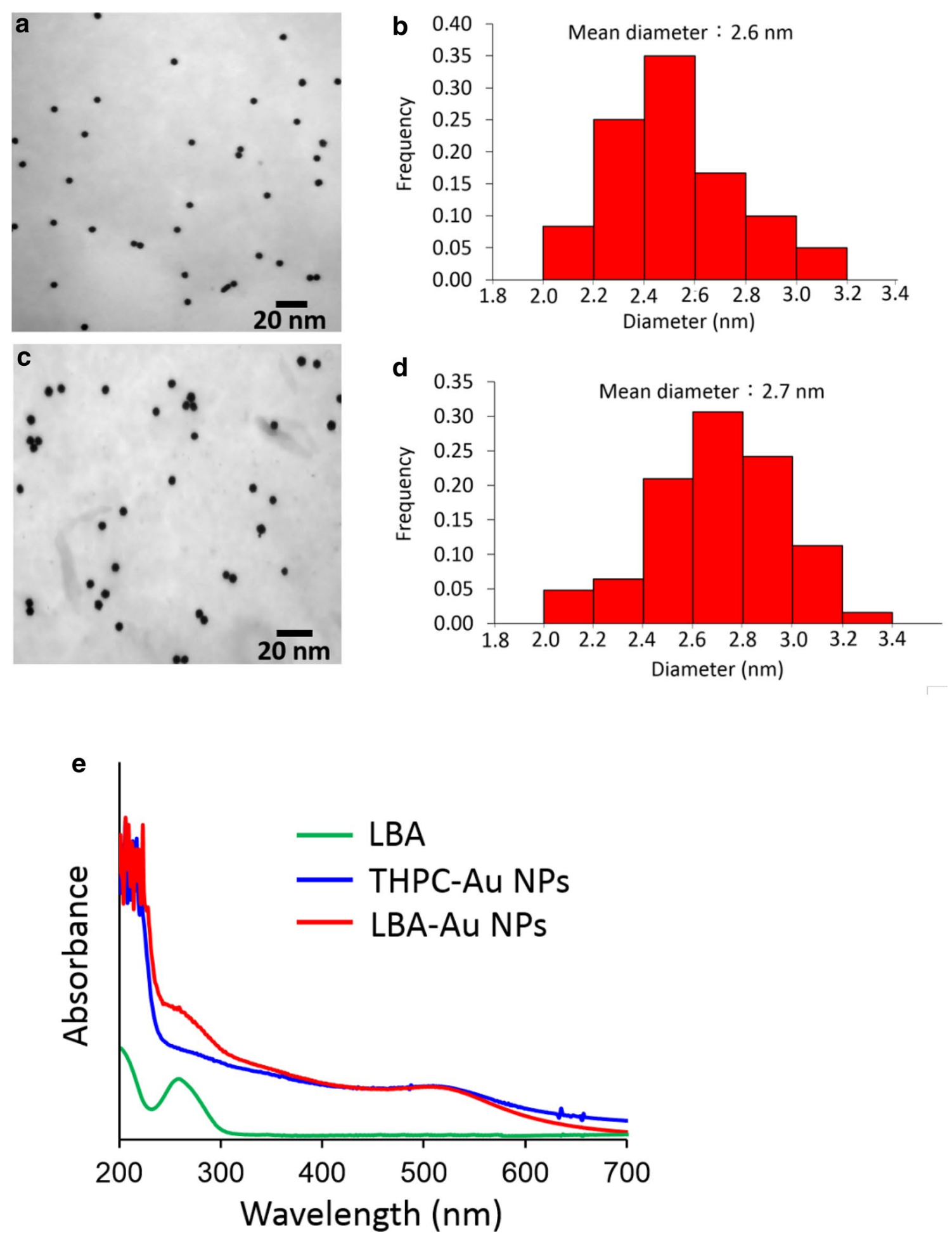

Fig. 1 TEM images of a THPC-Au NPs and $\mathbf{c} L B A-A u$ NPs. Size distribution histograms of (b) THPC-Au NPs and $\mathbf{d} L B A$-Au NPs. e Absorbance spectra obtained from LBAs, THPC-Au NPs, and LBA-Au NPs. The appearance of characteristic groups of LBAs and THPC-Au NPs observed in LBA-Au NPs supports the successful conjugation of LBAs on THPC-Au NPs 


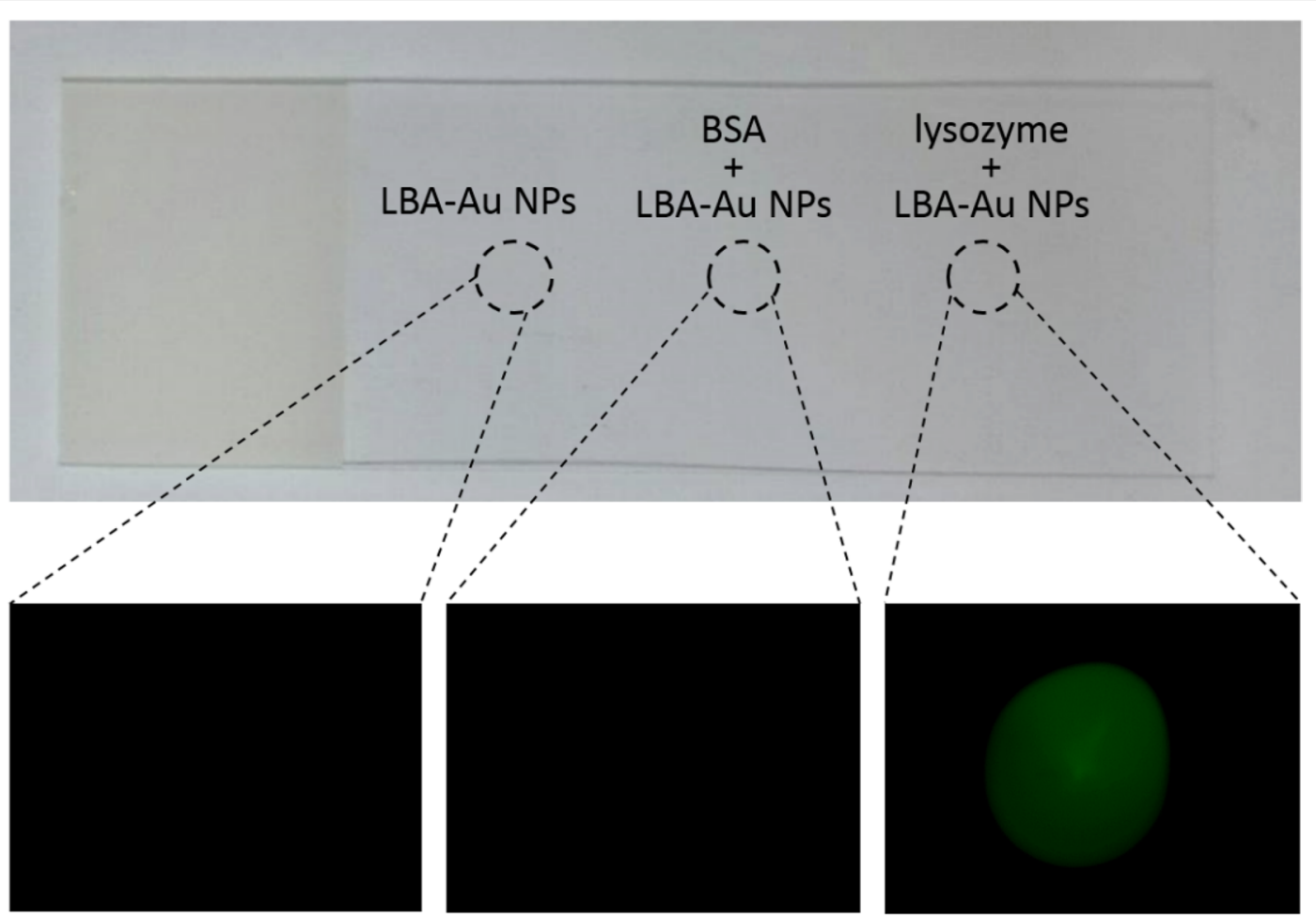

Fig. 2 Demonstration of lysozyme selectivity of LBA-Au NPs by fluorescence imaging. To achieve the fluorescent function of LBA-Au NPs, both Hex- (fluorescent molecule) and thiol-modified LBAs (5'-H S-TTTTTTATCAGGGCTAAAGAGTGCAGAGTTACTTAG-Hex-3') were used to replace thiol modified-LBAs in these experiments. The three regions defined by black dotted circles on the glass microscope slide (from left to right) were dropped with $10 \mu \mathrm{L}$ of aqueous solution respectively containing $10 \mu \mathrm{M}$ of PBS, bovine serum albumin (BSA), and lysozyme; the slide was then dried by air. Subsequently, the three regions were treated with LBA-Au NPs for $1 \mathrm{~h}$, followed by washing with deionized water to remove non-binding LBA-Au NPs. Finally, the region in each black dotted circle was observed using fluorescence microscopy. The left region (PBS-treated region) exhibited no fluorescence after treatment with THPC-Au NPs, indicating that THPC-Au NPs had no non-specific targeting on the glass microscope slide. No fluorescence was observed in the middle region (BSA-existed region) after treatment with LBA-Au NPs because LBA-Au NPs could not bind to BSA and were thus removed during washing. The right region (lysozyme-existed region) had an obvious fluorescence after treatment with LBA-Au NPS because of the specific lysozyme-targeting of the LBA-Au NPs. These results indicated that LBA-Au NPs still had excellent selectivity for lysozyme after the LBAs were conjugated on the surface of the Au NPs

resulting $\mathrm{Au}$ NPs with an average diameter of $14.1 \mathrm{~nm}$. The characteristic absorption band of the larger Au NPs is $\sim 530 \mathrm{~nm}$, which corresponds to particle size (Fig. 3d). Time-dependent spectra of the solution were recorded at $10 \mathrm{~s}$ intervals after mixing with $\mathrm{Au}^{+}$-complex solution and THPC-Au NPs (Fig. 3e). Results in Fig. 3e show the significantly increased intensities at a $530 \mathrm{~nm}$ wavelength every $10 \mathrm{~s}$, indicating that the sustained growth from $\mathrm{Au}$ seeds to larger Au NPs occurred over the course of $5 \mathrm{~min}$. Actually, the red color of the solution in Fig. 3a could be observed by the naked eye within $20 \mathrm{~s}$ after mixing with $\mathrm{Au}^{+}$-complex solution and THPC-Au NPs.
Figure 4 displays optical images of the rapid visualization of latent fingermarks collected using professional forensic tape after treatment with $\mathrm{Au}$ seed-mediated enhancement. Latent colourful collected immediately following deposition (Fig. 4a) and $24 \mathrm{~h}$ later (Fig. 4b) could be rapidly visualized and observed with the naked eye within $3 \mathrm{~min}$. The ridge patterns of fingermarks are clear and no background interference is observed. The visual ridge patterns were due to the larger sized Au NPs generated from the LBA-Au seeds bound to lysozyme in fingermarks through a seed growth process. To exclude the 
a

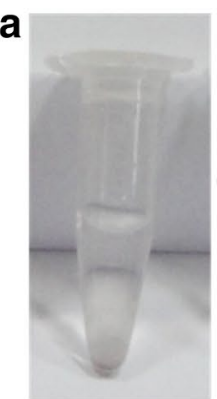

$\mathrm{Au}^{+}$-complex solution b

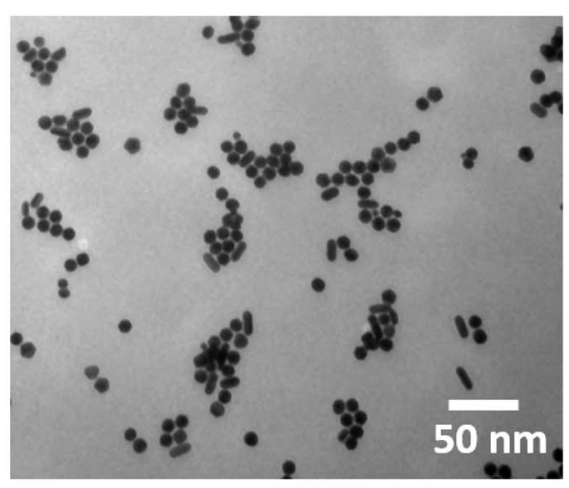

C
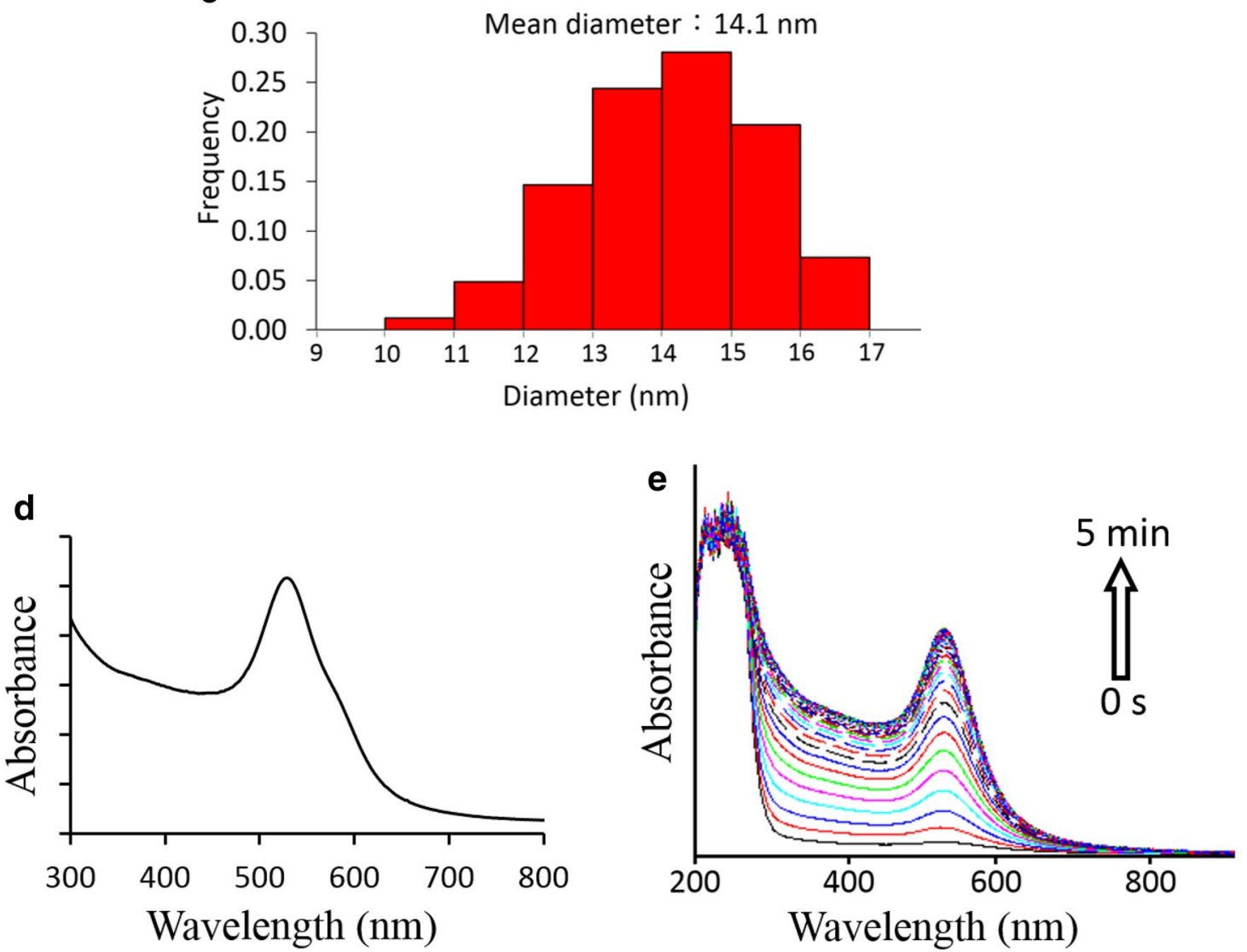

Fig. 3 a Photographs of $\mathrm{Au}^{+}$-complex solution taken $1 \mathrm{~min}$ after the addition of THPC-Au seeds (final ratio in solution: [Au $\left.{ }^{+}\right] /[\mathrm{Au}$ seed] $=152$ ). Final $\left[\mathrm{Au}^{+}\right]$and [Au seed] in solution were 1 and $6.6 \mathrm{nM}$, respectively; $\mathbf{b}$ TEM image of resulting lager Au NPs after mixing Au ${ }^{+}$-complex solution and THPC-Au NPs for 1 min; c Size distribution histograms of LBA-Au NPs; d Absorption spectrum of the resulting lager Au NPs in Fig. 2b; e Evolution of the absorption spectrum of the solution after mixing $\mathrm{Au}^{+}$-complex solution and THPC-Au NPs, where the spectra were recorded at intervals of $10 \mathrm{~s}$. Experimental conditions were the same as in Fig. $2 a$ 


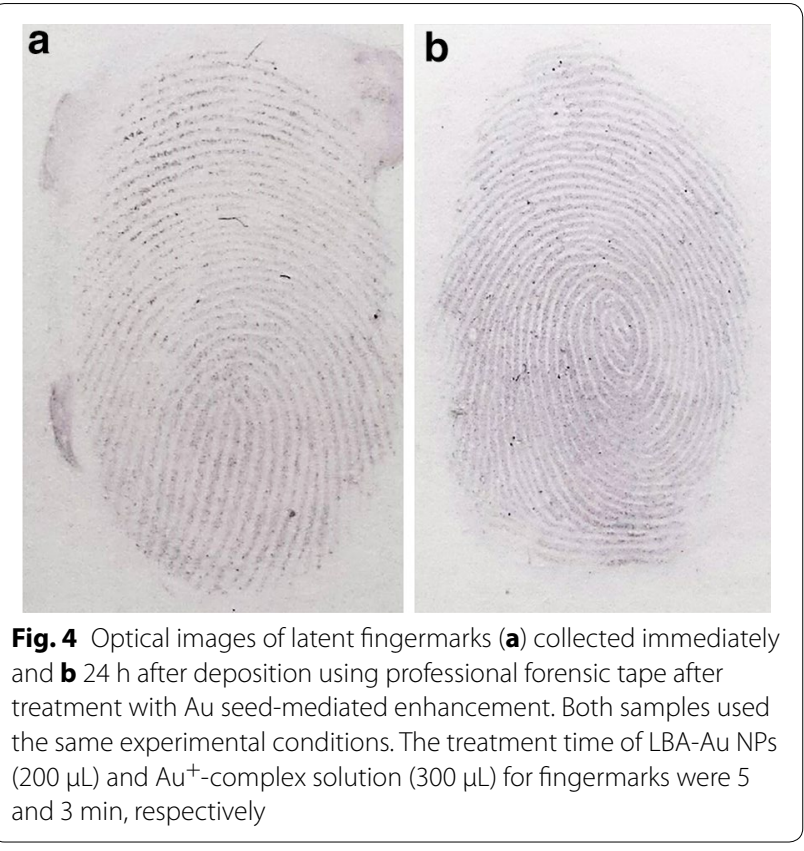

possibility of a new generation of Au NPs being generated from the $\mathrm{Au}^{+}$-complex solution, the collected fingermarks were directly treated with $\mathrm{Au}^{+}$-complex solution for $3 \mathrm{~min}$ (Fig. 5a). The fingermarks could not be detected because of the lack of Au seeds, thus the $\mathrm{Au}^{+}$of the $\mathrm{Au}^{+}-\mathrm{CTAB}$ complexes could not be reduced to $\mathrm{Au}^{0}$. To highlight the high Au seed-sensitivity in this method, THPC-Au NPs were designed to be replaced with LBA-Au NPs to first incubate latent fingermarks followed by treatment with the $\mathrm{Au}^{+}$-complex solution for $3 \mathrm{~min}$ (Fig. 5b). No fingermarks were visible on the tape because the THPC-Au NPs could not bind to lysozyme and were thus removed during the washing step, thus the $\mathrm{Au}^{+}-\mathrm{CTAB}$ complexes could not be reduced. However, when latent fingerprints collected using professional forensic tape (Fig. 5c) was first incubated with LBA-Au NPs and then treated with $\mathrm{Au}^{+}$complex solution, fingermarks were clearly visualized within 3 min and could be observed with the naked eye when collected immediately or $24 \mathrm{~h}$ following deposition. This rapid visualization can significantly reduce the amount of time required for fingermark collection and further post-treatment. Importantly, the stickiness of professional forensic tape barely caused any nonspecific adsorption of LBA-Au NPs and THPC-Au
NPs, and valleys between fingermark ridges could be clearly observed in each test.

To further prove the visual color of the ridges was due to the growth of Au NPs, the ridges of latent fingermarks and the backgrounds (valleys) between ridges were analysed by scanning electron microscopy (SEM) and energy-dispersive X-ray spectroscopy (EDX). Figure 6 shows no Au NPs or Au signals in the background, but many are observed in the ridges. The different sizes of the Au NPs were due to the selfcatalysis reaction occurring in the solid-liquid interfaces. These results also indicate that LBA-Au seeds have no non-specific binding and adsorption on the backgrounds but rather only target lysozymes in the ridges. Figure 7 shows a quantitative analysis of latent fingerprints in Fig. 6 after Au seed-mediated enhancement. A clear difference is found in the intensity of the ridges and backgrounds and the $\mathrm{p}$ value is less than 0.001 . The LBA-Au seed-targeted latent fingermarks are difficult to observe using SEM. To overcome this problem, we used 3-mercaptopropionic acid (MPA)coated $\mathrm{Si}$ wafers to prove the growth process from $\mathrm{Au}$ seeds to NPs. An MPA-coated Si wafer was incubated with a high concentration of THPC-Au seeds for easy observation in SEM, and then THPC-Au seeds were conjugated on the surface of $\mathrm{Si}$ wafers using a $\mathrm{Au}-\mathrm{S}$ bond, this is then followed by treatment with $\mathrm{Au}^{+}$CTAB complexes. Figure 8 shows the SEM images of an MPA-coated Si wafer treated with Au seed-mediated enhancement. The SEM image of the THPC-Au seed-treated MPA-coated Si wafer shows the Au seeds, but their small size leaves them unclear. Following treatment with $\mathrm{Au}^{+}-\mathrm{CTAB}$ complexes, the growth process from Au seeds to NPs was very obvious and many larger Au NPs were produced on the Si wafer. We monitored the entire process from Au seed conjugation on the Si wafer surface to the growth of Au seeds to NPs. This process was repeated using professional forensic tape.

Lysozyme is abundant in fingermark residues and is used here as a targeting biomolecule to visualize latent fingermarks. Although lysozyme is an enzyme, it can be stable in normal conditions. To demonstrate lysozyme tolerance under acidic and base solution conditions and at high temperatures, the collected latent fingermarks were pre-treated with $\mathrm{PBS}$ at $\mathrm{pH} 5$ and 9 and at 40 and $50{ }^{\circ} \mathrm{C}$ before treatment with $\mathrm{Au}$ seed-mediated 
a

\section{forensic tape}

b latent fingerprint

C latent fingerprint
$\stackrel{\mathrm{Au}^{+} \text {-complex solution }}{\stackrel{3}{3} \text { min }}$

$3 \mathrm{~min}$
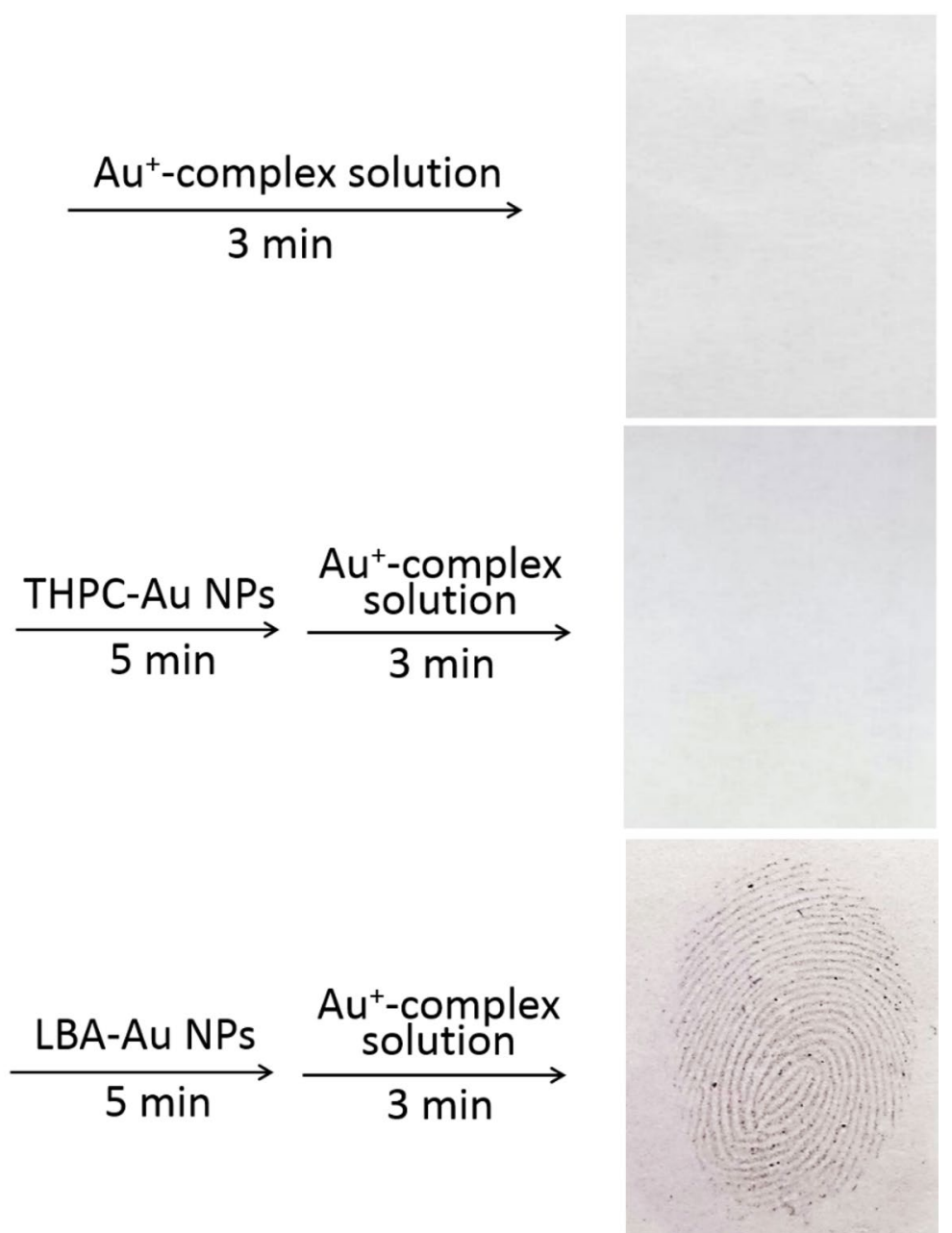

Fig. 5 Collected latent fingermarks were separately visualized under three different conditions: $\mathbf{a}$ treated with $\mathrm{Au}^{+}$-complex solution alone; $\mathbf{b}$ incubated with THPC-Au NPs first and then treated with $\mathrm{Au}^{+}$-complex solution; $\mathbf{c}$ incubated with LBA-Au NPs first and then treated with $\mathrm{Au}^{+}$-complex solution. All latent fingermarks $s$ were collected using professional-grade forensic tape. The used volumes of THPC-Au NPs and LBA-Au NPs were $200 \mu \mathrm{L}$ with particle concentrations of $132 \mu \mathrm{M}$. The used volumes of $\mathrm{Au}^{+}$-complex solution were $300 \mu \mathrm{L}$

enhancement. Figure 9a shows the results of $\mathrm{Au}$ seedmediated enhancement for the latent fingermarks after heating at 40 or $50{ }^{\circ} \mathrm{C}$ for $24 \mathrm{~h}$. The ridges of fingermarks were still clear with no apparent damage. This indicates that lysozymes could be stable under temperatures of up to $50^{\circ} \mathrm{C}$. Figure $9 \mathrm{~b}$ shows the results of Au seed-mediated enhancement for the latent fingermarks pre-incubated with PBS at $\mathrm{pH} 5$ and 9 for $24 \mathrm{~h}$. The ridges of fingermarks in both conditions (pH 5 and 9) were also clear, though the ridges of those prepared at pH 5 are relatively sharper, possibly because fewer lysozymes were removed by the $\mathrm{pH} 9$ solution.

In addition to using professional forensic tape, Fig. 10a, $\mathrm{b}$ respectively show the Au seed-mediated enhanced visualization of latent fingermarks using plastic and glass (see Fig. 10a). Ridge contrast intensity was found to be related to the use of different composites used in the $\mathrm{CD}$ surface of various commercial products. $\mathrm{Au}$ 

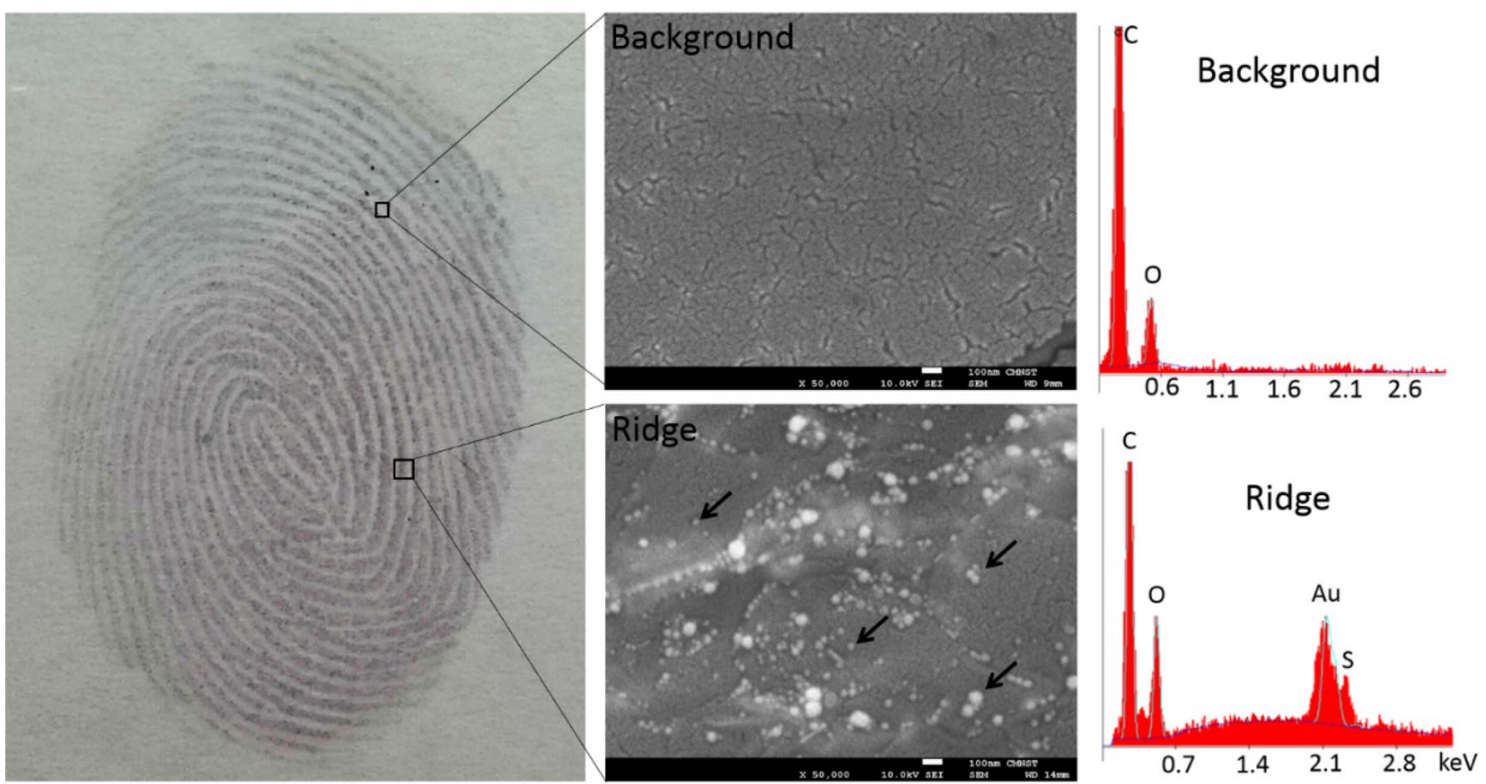

Fig. 6 SEM images and EDX analyses of the ridges of fingermarks and the backgrounds between ridges after collected latent fingermarks were incubated with LBA-Au NPs first and then treated with $\mathrm{Au}^{+}$-complex solution. No signals of Au and Au NPs were detected and observed in the backgrounds between ridges of fingermarks, but Au NPs (black arrows) with different sizes appeared in the ridges of fingermarks and EDX proved their component was Au. These results indicated the visual ridges of fingermarks were certainly due to the growth of Au NPs from Au seeds. This latent fingermark was collected using professional-grade forensic tape. The used volumes of THPC-Au NPs and LBA-Au NPs were $200 \mu \mathrm{L}$ with particle concentrations of $132 \mu \mathrm{M}$. The used volumes of $\mathrm{Au}^{+}$-complex solution were $300 \mu \mathrm{L}$. Scale bars in both SEM images are $100 \mathrm{~nm}$

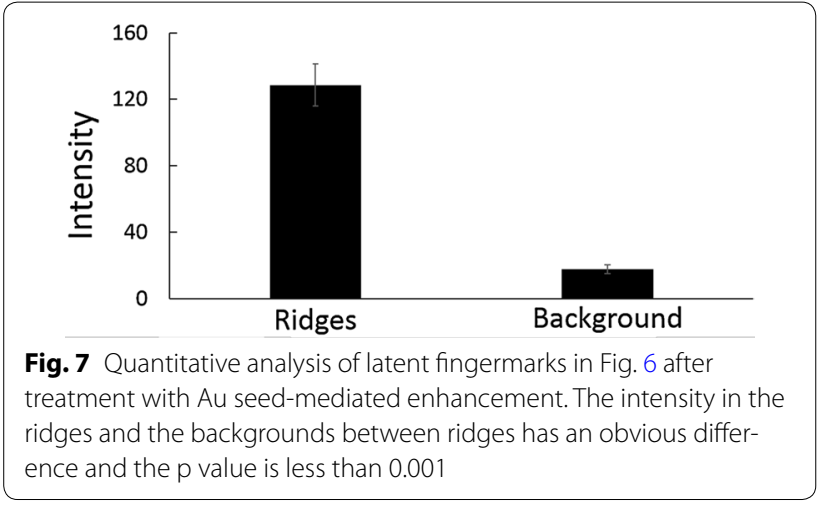

seed-mediated enhancement is not effective for visualizing latent fingermarks on porous substrates such as paper which adsorbs the aqueous working solution.
The cyanoacrylate and triketohydrindene hydrate-based methods may fail to visualize latent fingermarks then the primary amine groups of proteins are completely consumed. In contrast, in Au seed-mediated enhancement, the growth of Au NPs can be easily sustained through the addition of more $\mathrm{Au}^{+}-\mathrm{CTAB}$ solution, allowing this method to work effectively even with smaller amounts of available proteins. The greatest difference between $\mathrm{Au}^{+}$$\mathrm{CTAB}$ complexes and the silver staining solution used in iMMD is the image contrast between the ridges of fingermarks and background. For silver staining solution, longer incubation times will produce a significant background, reducing the visual contrast between the ridges and the underlying substrate. However, longer incubation using the $\mathrm{Au}^{+}$-CTAB complex produces a sharper image contrast between the ridges and the underlying substrate. The $\mathrm{Au}^{+}-\mathrm{CTAB}$ complexes only reacted with $\mathrm{Au}$ seeds 


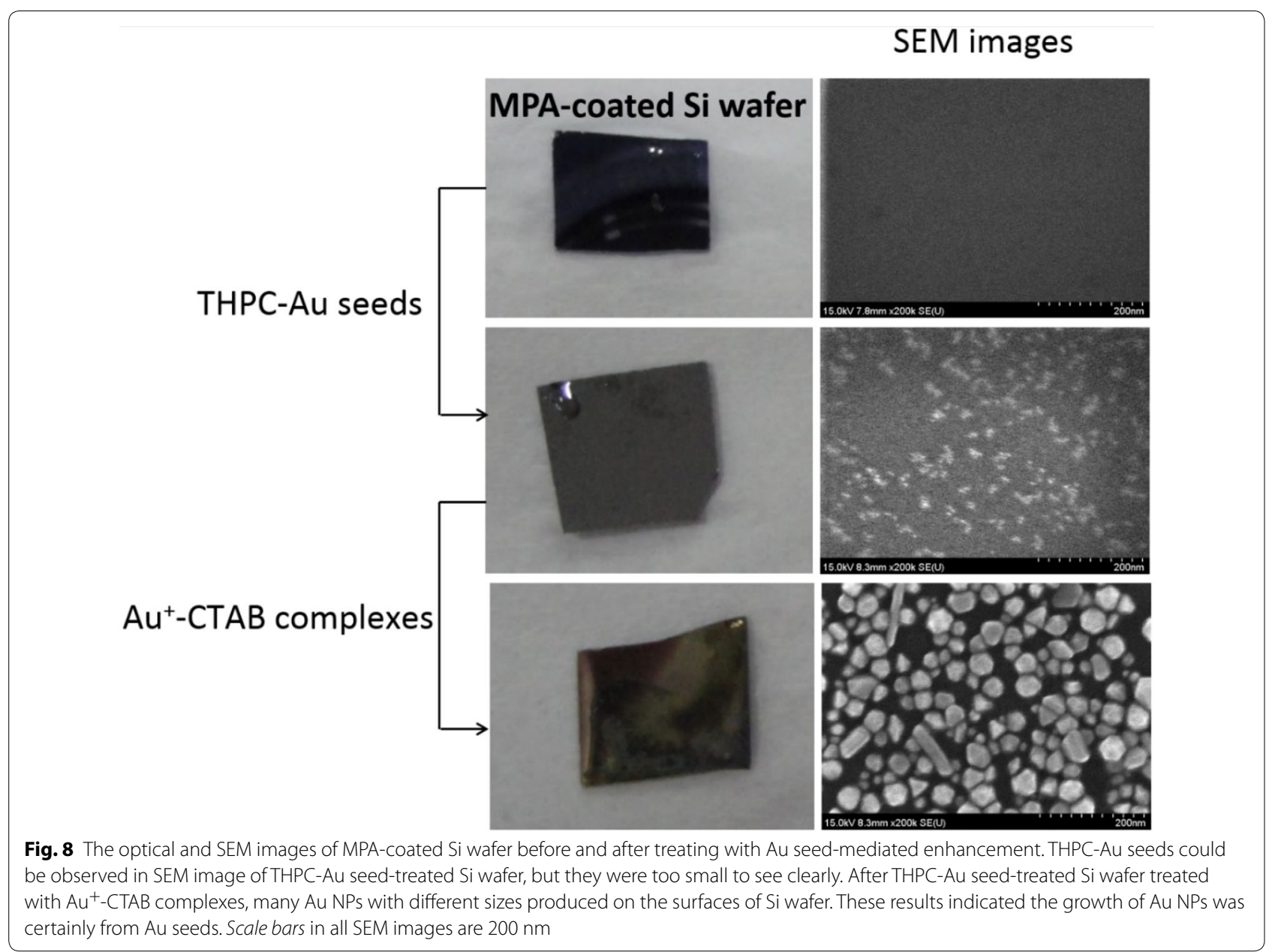

and then reduced from $\mathrm{Au}^{+}$to $\mathrm{Au}^{0}$ on the surface of the $\mathrm{Au}$ seeds. This self-catalysis process produced no background interference even with extended incubation times. In addition to incubation time, image contrast was also a function of solution concentrations. Using highly concentrated $\mathrm{Au}^{+}$or $\mathrm{Ag}^{+}$solutions might decrease the time required to enhance image contrast. However, too fast a reduction process can increase background interference. Using solutions in large volume could enhance the image contrast, but this requires additional time. Thus, both conditions (high concentrations and large volumes) easily produce background interference in the silver staining solution method, but no such interference occurs using the $\mathrm{Au}^{+}-\mathrm{CTAB}$ complex. Figure 11 shows fingermark images after treatment following long incubation times or high concentrations of the LBA-Au NPs or $\mathrm{Au}^{+}$-complex solutions. Increasing the LBA-Au NP incubation time from $5 \mathrm{~min}$ to 15 or $30 \mathrm{~min}$ (Fig. 11a), does not noticeably degrade the fingermark images because the originally used concentration $(132 \mu \mathrm{M})$ of LBA-Au NPs is sufficient to bind specifically to fingermark lysosomes, and binding reaches saturation within $5 \mathrm{~min}$, thus additional incubation time will not result in significant additional binding. Increasing the concentrations of LBA-Au NPs increased from $132 \mu \mathrm{M}$ to 264 or $396 \mu \mathrm{M}$ (Fig. 11b) also failed to significantly change the results. Treating latent fingermarks treated with 264 or $396 \mu \mathrm{M}$ of LBA-Au NPs produced deeper colors, representing the additional Au content on the fingermarks. Another possible reason is that higher concentrations of LBA-Au NPs can further increase the binding amount of LBA-Au NPs on the lysozymes. Importantly, neither increased incubation time nor LBA-Au NP 

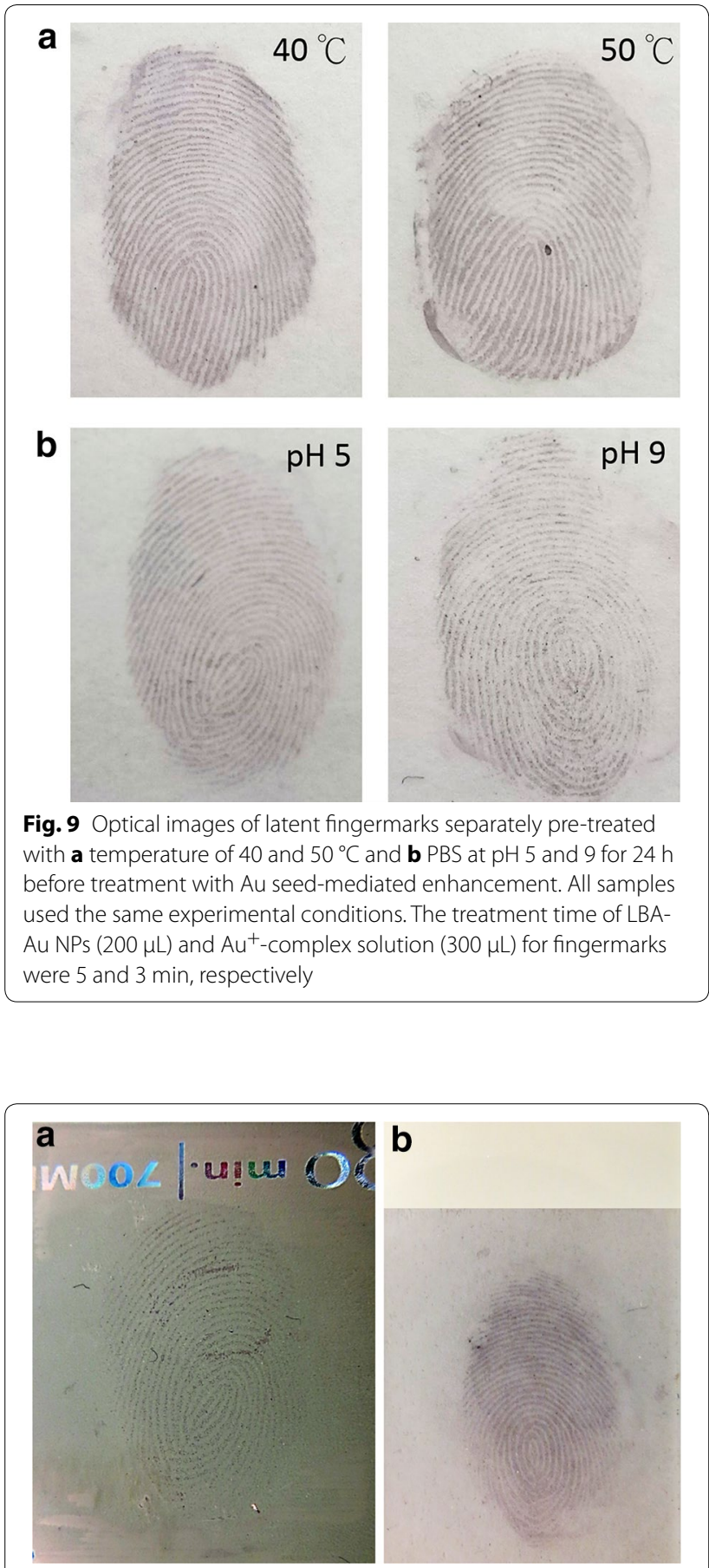

Fig. 10 Optical images of using Au seed-mediated enhancement to visualize the latent fingermarks on $\mathbf{a}$ plastic and $\mathbf{b}$ glass. The plastic is the surface of commercial CD. The glass is general glass slide. Both samples used the same experimental conditions. The treatment time of LBA-Au NPs $(200 \mu \mathrm{L})$ and $\mathrm{Au}^{+}$-complex solution $(300 \mu \mathrm{L})$ for fingermarks were 5 and 3 min, respectively concentrations produced obvious background interference in Fig. 11a, b. Similarly, increasing the incubation time of $\mathrm{Au}^{+}$-complex solutions from $5 \mathrm{~min}$ to 15 or $30 \mathrm{~min}$ (Fig. 11c) still produced clear fingermark images with no obvious background interference. Increasing the concentration of $\mathrm{Au}^{+}$-complex solutions from $470 \mu \mathrm{M}$ to 940 or $1410 \mu \mathrm{M}$ (Fig. 11d) produces fingermark images with more pronounced color because $\mathrm{Au}^{+}$-complexs are consumptive substrates and can be reduced on the surface of the Au seeds until they are completely exhausted. Importantly, despite the pronounced fingermark colors in Fig. 11d, no obvious background interference is observed. These results clearly show that background interference is not increased by increasing the incubation time or the concentration of LBA-Au NPs or $\mathrm{Au}^{+}$-complex solutions in Au seed-mediated enhancement. All fingermarks are different, even those taken from different fingers from a single individual. In fact, the visualizing of latent fingermarks usually involves adding more solution or lengthening the incubation period until the marks appear on the substrates. In this situation, Au seed-mediated enhancement is more effective than the silver staining solution to obtain good image contrast without background interference.

\section{Conclusions}

In conclusion, we report a simple, inexpensive, and fast method for the rapid visualization of latent fingermarks on the non-porous substrates using Au seed-mediated enhancement. LBA-Au NPs were used as Au seeds with a $\mathrm{Au}^{+}$-complex solution composed of $\mathrm{HAuCl}_{4}, \mathrm{CTAB}$, and $\mathrm{AA}$ used as a growth agent. The $\mathrm{Au}^{+}$ion of the $\mathrm{Au}^{+}$$\mathrm{CTAB}$ complexes could be only reduced from $\mathrm{Au}^{+}$to $\mathrm{Au}^{0}$ in the presence of the Au seeds. Importantly, the procedure requires only two main steps for latent fingermark visualization and can be completed in less than $10 \mathrm{~min}$. In the first step, latent fingermarks are incubated with LBA$\mathrm{Au}$ NPs for $5 \mathrm{~min}$. In the second step, the latent fingermarks are treated with the $\mathrm{Au}^{+}-\mathrm{CTAB}$ complex solution for $3 \mathrm{~min}$. In fact, latent fingermarks can be visualized for observation in less than 3 min after treatment with $\mathrm{Au}^{+}$-complex solution and without background interference. The proposed approach offers faster detection and visualization of latent fingermarks than existing methods. Moreover, the results are observable directly with the naked eye, without the use of expensive or sophisticated instruments. The proposed method is expected to increase detection efficiency for latent fingermarks, thus reducing time requirements and costs for forensic investigations and medical diagnostics. 


\section{Used conditions in this study}

LBA-Au NPs $-\left[\begin{array}{l}\text { Concentration : } 132 \mu \mathrm{M} \\ \text { (particle conc.) } \\ \text { Volume : } 200 \mu \mathrm{L} \\ \text { Incubation time }: 5 \mathrm{~min}\end{array}\right.$

a Incubation time of LBA-Au NPs
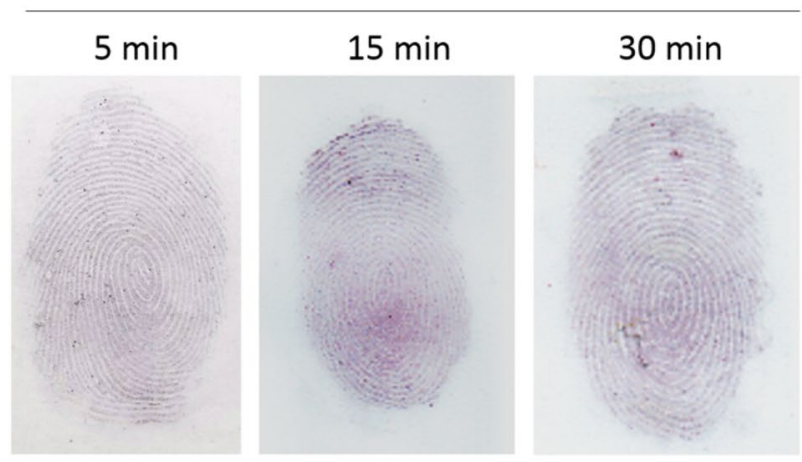

C

Incubation time of $\mathrm{Au}^{+}$-complex solution

$5 \mathrm{~min}$

$15 \mathrm{~min}$
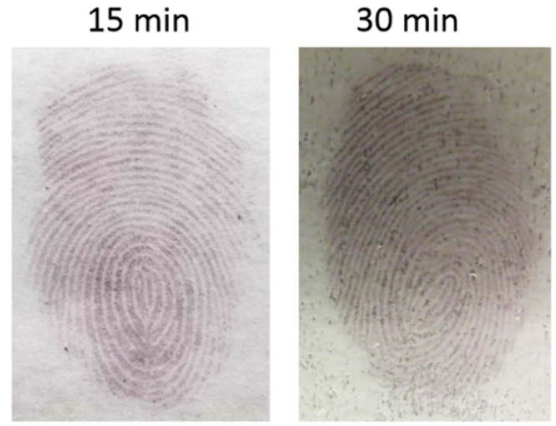

b

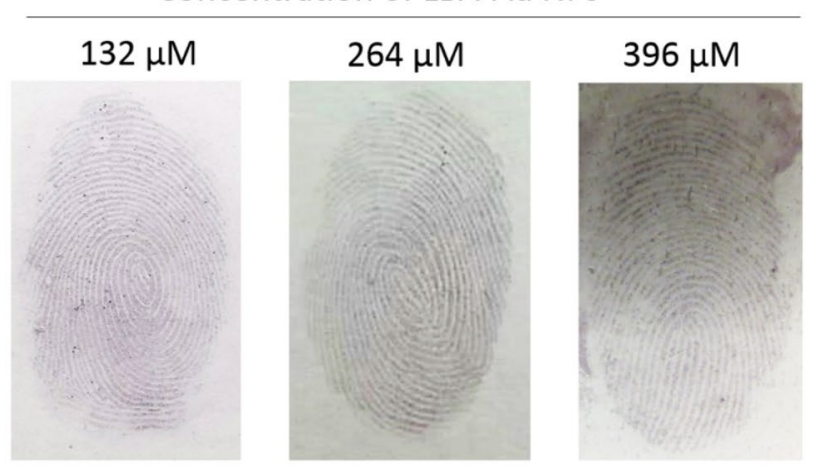

d Concentration of $\mathrm{Au}^{+}$-complex solution

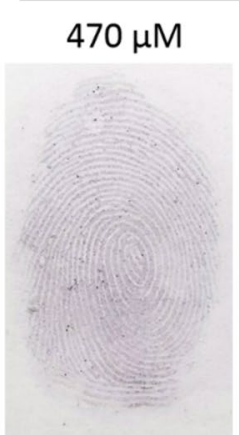

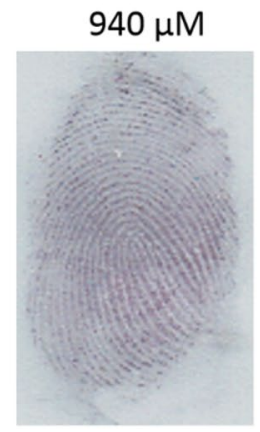

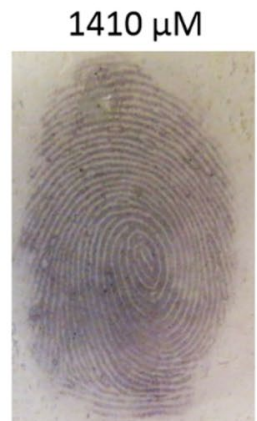

Fig. 11 Optical images of using Au seed-mediated enhancement to visualize the latent fingermarks at different conditions with increasing: a the incubation time (5, 15, and $30 \mathrm{~min})$ of LBA-Au NPs, $\mathbf{b}$ the concentration (132, 264, and $396 \mu \mathrm{L})$ of LBA-Au NPs, c the incubation time (5, 15, and $30 \mathrm{~min}$ ) of $\mathrm{Au}^{+}$-complex solution and $\mathbf{d}$ the concentration $(132,264$, and $396 \mu \mathrm{L})$ of $\mathrm{Au}^{+}$-complex solution. The upper frame means that the used conditions in this study. In a-d tests, only one parameter is changed in each test keeping the rest of the conditions analogous to the upper frame

\section{Authors' contributions}

Study design: FYC. Data collection: CHS, CCY, FYC. Drafting manuscript: FYC. All the authors reviewed and approved the final manuscript. All authors read and approved the final manuscript.

\section{Author details}

${ }^{1}$ Institute for Translational Research in Biomedicine, Kaohsiung Chang Gung Memorial Hospital, Kaohsiung 833, Taiwan. ${ }^{2}$ Department of Biomedical Imaging and Radiological Sciences, National Yang Ming University, Taipei 112 Taiwan. ${ }^{3}$ Department of Chemistry, Chinese Culture University, 55, Hwa-Kang Road, Yang-Ming-Shan, Taipei 11114, Taiwan.

\section{Acknowledgements}

This work was supported by Grant MOST 103-2113-M-006-005,

103-2633-B-182A-001, 103-2320-B-182A-004, and 103-2320-B-182A-004 -MY3 from the Taiwan Ministry of Science and Technology and CMRPG8E1461 from Kaohsiung Chang Gung Memorial Hospital, Taiwan.

\section{Competing interests}

The authors declare that they have no competing interests.

Received: 1 October 2016 Accepted: 13 November 2016

Published online: 25 November 2016

\section{References}

1. Faulds H. On the skin-furrows of the hand. Nature. 1880;22:605.

2. Lee HC, Gaensslen RE. Advances in fingerprint technology. 2nd ed. Boca Raton: CRC; 2001

3. Herschel WJ. Skin furrows of the hand. Nature. 1880:23:76

4. Polson CJ. Finger prints and finger printing. J Crim Law Criminol. 1950;41:495-517 
5. Li K, Qin W, Li F, Zhao X, Jiang B, Wang K, Deng S, Fan C, Li D. Nanoplasmonic imaging of latent fingerprints and identification of cocaine. Angew Chem Int Ed. 2013;52:11542-5.

6. Hazarika $P$, Jickells $S M$, Wolff $K$, Russell DA. Imaging of latent fingerprints through the detection of drugs and metabolites. Angew Chem Int Ed. 2008:47:10167-70.

7. Hazarika P, Jickells SM, Wolff K, Russell DA. Multiplexed detection of metabolites of narcotic drugs from a single latent fingermark. Anal Chem. 2010;82:9150-4

8. Leggett R, Lee-Smith EE, Jickells SM, Russell DA. "Intelligent" fingerprinting: simultaneous identification of drug metabolites and individuals by using antibody-Functionalized nanoparticles. Angew Chem Int Ed. 2007:46:4100-3.

9. Spindler X, Hofdtetter O, McDonagh AM, Roux C, Lennard C. Enhancement of latent fingermarks on non-porous surfaces using anti-l-amino acid antibodies conjugated to gold nanoparticles. Chem Commun. 2011;47:5602-4.

10. Miao D, Jain AK. Handbook of fingerprint recognition. 2nd ed. Amsterdam: Springer; 2009.

11. Choi MJ, McDonagh AM, Maynard P, Roux C. Metal-containing nanoparticles and nano-structured particles in fingermark detection. Forensic Sci Int. 2008;179:87-97.

12. Sameband M, Shweky I, Banin U, Mandler D, Alomg J. Application of nanoparticles for the enhancement of latent fingerprints. Chem Commun. 2007;11:1142-4.

13. Becue A, Champod C, Margot P. Use of gold nanoparticles as molecular intermediates for the detection of fingermarks. Forensic Sci Int. 2007;168:169-76

14. Wood M, Maynard P, Spindler X, Lennard C, Roux C. Visualization of latent fingermarks using an aptamer-based reagent. Angew Chem Int Ed. 2012;51:12272-4.

15. Shan $X$, Patel U, Wang $S$, Iglesias $R$, Tao N. Imaging local electrochemical current via surface plasmon resonance. Science. 2010;327:1363-6.

16. Xu L, Zhou Z, Zhang C, He Y, Su B. Electrochemiluminescence imaging of latent fingermarks through the immunodetection of secretions in human perspiration. Chem Commun. 2014;50:9097-100.

17. Ma R, Bullock E, Maynard P, Reedy B, Shimmon R, Lennard C, Roux C, McDonagh A. Fingermark detection on non-porous and semi-porous surfaces using $\mathrm{NaYF}_{4}: \mathrm{Er}, \mathrm{Yb}$ up-converter particles. Forensic Sci Int. 2011;207:145-9.
18. Wang J, Wei T, Li X, Zhang B, Wang J, Huang C, Yuan Q. Near-infrared-lightmediated imaging of latent fingerprints based on molecular recognition. Angew Chem Int Ed. 2014;53:1616-20.

19. He Y, Xu L, Zhu Y, Wei Q, Zhang M, Su B. Immunological multimetal deposition for rapid visualization of sweat fingerprints. Angew Chem Int Ed. 2014;53:12609-12.

20. Phillips DC. The hen egg-white lysozyme molecule. Proc Natl Acad Sci USA. 1967;57:483-95.

21. Hartzell-Baguley B, Hipp RE, Morgan NR, Morgan SL. Chemical composition of latent fingerprints by gas chromatography-mass spectrometry. An experiment for an instrumental analysis course. J Chem Educ. 2007:84:689.

22. Marshall T. Analysis of human wweat proteins by two-dimensional electrophoresis and ultrasensitive silver staining. Anal Biochem. 1984;139:506-9.

23. Pham T, Jackson JB, Halas NJ, Lee TR. Preparation and characterization of gold nanoshells coated with self-assembled monolayers. Langmuir. 2002:18:4915-20.

24. Jana NR, Gearheart L, Murphy CJ. Evidence for seed-mediated nucleation in the chemical reduction of gold Salts to gold nanoparticles. Chem Mater. 2001;13:2313-22.

25. Esumi K, Matsuhisa K, Torigoe K. Preparation of rodlike gold particles by UV irradiation using cationic micelles as a template. Langmuir. 1995;11:3285-7.

26. Rodriguez-Fernandez J, Perez-Juste J, Mulvaney P, Liz-Marzan LM. Spatially-directed oxidation of gold nanoparticles by $\mathrm{Au}(\mathrm{III})-\mathrm{CTAB}$ complexes. J Phys Chem B. 2005;109:14257-61.

27. Perez-Juste J, Liz-Marzan LM, Carnie S, Chan DYC, Mulvaney P. Electricfield-directed growth of gold nanorods in aqueous surfactant solutions. Adv Funct Mater. 2004;14:571-9.

28. Jana NR, Gearheart L, Murphy CJ. Seeding growth for size control of 5-40 nm diameter gold nanoparticles. Langmuir. 2001;17:6782-6.

29. Li C, Fan F, Yin B, Chen L, Ganguly T, Tian Z. Au+ -cetyltrimethylammonium bromide solution: a novel precursor for seed-mediated growth of gold nanoparticles in aqueous solution. Nano Research. 2013;6:29-37.

\section{Submit your next manuscript to BioMed Central and we will help you at every step:}

- We accept pre-submission inquiries

- Our selector tool helps you to find the most relevant journal

- We provide round the clock customer support

- Convenient online submission

- Thorough peer review

- Inclusion in PubMed and all major indexing services

- Maximum visibility for your research

Submit your manuscript at www.biomedcentral com/submit 